Nitroreduktase aktiv sind, während das TPNH allein auf die Azoreduktase einwirkt.

Im ersten Falle käme es folglich zu einer größeren verfügbaren $\mathrm{H}$-Menge, wobei die aromatische Nitrogruppe als aspezifischer Akzeptor wirken dürfte.

Bezüglich der Flavine, mittels welcher der Transport von Elektronen von den reduzierten Pyridinnucleotiden zum Substrat hin erfolgt, könnte vielleicht das Nitroreduktase-System ihrer Abnahme in der Leber gegenüber weniger empfindlich sein, und zwar durch die Möglichkeit einer stärkeren Ausnützung der Eigenschaft der
Flavine selbst, als künstliche Träger von Elektronen in freier Form, also nicht an das Enzym gebunden, zu wirken.

In diesem Zusammenhange soll endlich daran erinnert werden, daß das Nitroreduktase-System der Säugetierzellen grundsätzlich von jenem verschieden ist, das von $S_{A Z}$ und $S_{L I E}{ }^{4,5}$ für die Bakterienzellen beschrieben wurde.

${ }^{4}$ A. K. SAz u. R. B. Stre, Arch. Biochem. Biophysics 5l, 5 [1954]

5 A. K. SAZ u. R. B. SLIE, J. biol. Chemistry 210, 407 [1954].

\section{Weiterentwicklung der manometrischen Methoden}

\section{(Herstellung definierter Blausäure-Konzentrationen)}

Von Hans-Siegried Gewitz und Wolfgang Völker

Aus dem Max-Planck-Institut für Zellphysiologie, Berlin-Dahlem

(Z. Naturforschg. 15 b, 625 [1960] ; eingegangen am 7. Juli 1960)

Die Blausäure wird in den Manometriegefäßen nach beendeter Gasdurch. leitung und nach dem Schließen der Hähne aus Quecksilbercyanid entwickelt.

Da Blausäure flüchtig ist, und also zugesetzte Blausäure bei manometrischen Versuchen während des Gas. durchleitens ausgetrieben wird, war die Herstellung definierter Blausäure-Konzentrationen bei der Manometrie bisher im allgemeinen nicht möglich. Mit Hilfe der neuen Wannengefäße ${ }^{1}$ (Abb. 1) kann dem auf folgende Weise abgeholfen werden.

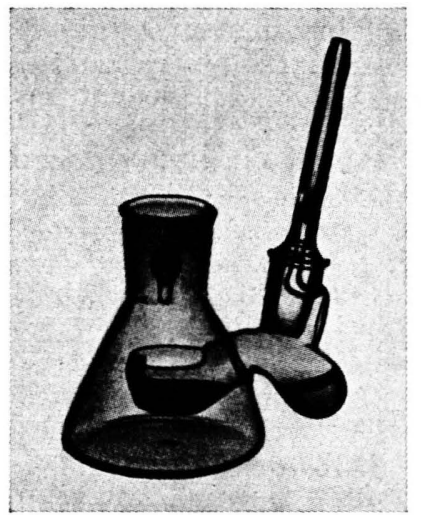

Abb. 1. Wannengefäß. $0,2 \mathrm{~cm}^{3} \quad \mathrm{Hg}(\mathrm{CN})_{2}$-Lösung in der Wanne, 0,2 cm $\mathrm{cm}^{3}$ 10-proz. NaCl-Lösung in 5-n. $\mathrm{H}_{2} \mathrm{SO}_{4}$ in der mit der Wanne verbundenen Birne, im Hauptraum $\sim 3 \mathrm{~cm}^{3}$ Zellsuspension.

Wir geben in die Wanne eine wäßrige Lösung von Quecksilbercyanid (das unter den Bedingungen der Manometrie nicht flüchtig und nicht dissoziiert ist), in

1 O. WARBURG u. G. KRIPPAHL, Z. Naturforschg. 14 b, 561 [1959]. die mit der Wanne verbundene Birne eine stark schwefelsaure Kochsalzlösung, leiten (beliebig lange) Gas durch die Manometriegefäße, schließen die Hähne und geben erst dann, nach beendeter Gasdurchleitung und nach Schließen der Hähne, die saure Kochsalzlösung aus der Birne in die Wanne, wobei durch die Reaktion:

$$
\mathrm{Hg}(\mathrm{CN})_{2}+2 \mathrm{HCl}=\mathrm{Hg}(\mathrm{Cl})_{2}+2 \mathrm{HCN}
$$

die Blausäure in die Manometriegefäße entwickelt wird. Nach der Einstellung des Verteilungsgleichgewichts, befindet sich dann der größte Teil der Blausäure im Hauptraum, falls das Flüssigkeitsvolumen im Hauptraum groß ist gegen das Flüssigkeitsvolumen in der Wanne.

Denn ist $a$ das Flüssigkeitsvolumen im Hauptraum und $b$ das Flüssigkeitsvolumen in der Wanne + Birne und werden $x \mu$ Mole $\mathrm{CN}$ als $\mathrm{Hg}(\mathrm{CN})_{2}$ in die Wanne gegeben, so befinden sich nach Einstellung des Verteilungsgleichgewichts

$$
x \cdot \frac{a}{a+b} \mu \text { Mole HCN }
$$

in $a$ im Hauptraum. Die Konzentration der Blausäure in $a$ im Hauptraum beträgt dann

$$
c=\frac{x}{a+b}\left[\frac{\mu \text { Mole HCN }}{\mathrm{cm}^{3}}\right] .
$$

Der Bunsensche Absorptionskoeffizient der Blausäure ${ }^{2}$ ist bei $20^{\circ} \alpha=240$, so daß bei dieser Rechnung die HCN im Gasraum vernachlässigt werden kann.

Zum Beispiel waren die Volumina und Substanzmengen:

Flüssigkeitsvolumen im Hauptraum $4,0 \mathrm{~cm}^{3}$ (Wasser), Flüssigkeitsvolumen in der Wanne $0,2 \mathrm{~cm}^{3} \frac{m}{50}-\mathrm{Hg}$ $(\mathrm{CN})_{2}=4,0 \mu$ Mole $\mathrm{Hg}(\mathrm{CN})_{2}$,

Flüssigkeitsvolumen in der Birne $0,2 \mathrm{~cm}^{3}(10 \% \mathrm{NaCl}$, 5-n. $\mathrm{H}_{2} \mathrm{SO}_{4}$ ).

Nach Einkippen der sauren NaCl-Lösung in die Wanne und 10 Minuten Schütteln ergab die Titration der HCN in $a$ im Hauptraum (nach L i e b i g mit Silbernitrat und KJ) $7,1 \mu$ Mole HCN, während nach (1) berechnet waren

$$
8 \cdot \frac{4}{4+0,4}=7,26 \mu \text { Mole HCN. }
$$

2 O. W 